\title{
MILLIMETER-GAP MAGNETICALLY INSULATED TRANSMISSION LINE POWER FLOW EXPERIMENTS
}

\author{
B. T. Hutsel ${ }^{\xi}$, B. S. Stoltzfus, E. W. Breden, W. E. Fowler, P. A. Jones, D. W. Justus, \\ F. W. Long, D. J. Lucero, K. A. MacRunnels, M. G. Mazarakis, J. L. Mckenney, \\ J. K. Moore, T. D. Mulville, J. L. Porter, M. E. Savage, W. A. Stygar \\ Sandia National Laboratories, PO Box 5800, Mail Stop 1194 \\ Albuquerque, NM, USA
}

\section{Abstract}

An experiment platform has been designed to study vacuum power flow in magnetically insulated transmission lines (MITLs). The platform is driven by the Mykonos-V LTD accelerator to drive a coaxial MITL with a millimeter-scale anode-cathode gap. The experiments conducted quantify the current loss in the MITL with respect to vacuum pumpdown time and vacuum pressure. MITL gaps between $1.0 \mathrm{~mm}$ and $1.3 \mathrm{~mm}$ were tested. The experiment results revealed large differences in performance for the 1.0 and $1.3 \mathrm{~mm}$ gaps. The $1.0 \mathrm{~mm}$ gap resulted in current losses of $40 \%-60 \%$ of the peak current. The $1.3 \mathrm{~mm}$ gap resulted in current losses of less than $5 \%$ of peak current. Classical MITL models that neglect plasma expansion predict that there should be zero current loss, after magnetic insulation is established, for both of these gaps.

\section{I.INTRODUCTION}

Magnetically insulated transmission lines (MITLs) are commonly used in the final stages of pulsed power systems to transfer power at high voltage and current to the physics-package load. Areas such as the convolute and inner-MITL of the Z-Machine require $\mathrm{MA} / \mathrm{cm}$ level currents to be transmitted with vacuum gaps of $1 \mathrm{~cm}$ or less [1]. Future pulsed power systems, which will deliver greater power to loads, will require MITLs to transfer power at greater power densities. Understanding and minimizing current loss within the MITL will be a critical design issue for these larger pulsed power systems.

MITL power flow has been studied in detail. In order to support MITL design and understand current loss in a MITL, many individual studies involving simulation [24], analytic calculations [5-8], and experiment [9-14] have been published.

In these experiments, the power flow in a low lineal current density (10's of kA/cm), millimeter-scale MITL gap was studied. The experiments were conducted at low lineal current density to minimize the influence of anode heating and ion emission from anode surfaces [15]. The experiments performed were directed towards studying the effect of vacuum conditions on the current loss in a MITL. It is known that surface contamination alters the emission characteristics of electrodes [16, 17]. In the $Z$ MITL system, it has been observed that increased vacuum pumpdown times reduces the partial pressures of electrode-surface contaminants and can reduce current loss across the MITL anode-cathode (AK) gap [18]. The experiments described in this report investigated the MITL current loss with respect to vacuum pressure in a MITL in a controlled manner, without the variability of different machine configurations or complex dynamic experiment loads that are used on $\mathrm{Z}$.

\section{II.EXPERIMENT SETUP}

\section{A. Pulsed Power Driver}

The Mykonos LTD module served as the pulsed power driver for the vacuum transmission line power flow experiments. The vacuum transmission line power flow tests were the first experiments to be completed on an upgraded five-cavity Mykonos LTD module (Mykonos V).

The Mykonos V LTD module is made up of five, threemeter diameter LTD cavities. Each cavity consists of 36 LTD bricks, each built with a HCEI $200 \mathrm{kV}$ multi-gap switch [19] and two $40 \mathrm{nF}$ General Atomics 31165 capacitors. The five cavities are triggered sequentially and drive a matched impedance coaxial water transmission line. The cavities are triggered with a 6.6 ns delay between cavities to match the transit time of the power pulse in the water transmission line. The cavities and water transmission line are shown in Figure 1. The LTD cavities are designed to operate at up to $+/-100 \mathrm{kV}$ charge voltage. At full charge voltage, Mykonos $\mathrm{V}$ nominally produces a $1 \mathrm{MA}, 500 \mathrm{kV}$ pulse with a rise time $(10 \%$ $90 \%$ ) of $60 \mathrm{~ns}$ and a pulse width (FWHM) of $160 \mathrm{~ns}$ into a $0.5 \mathrm{ohm}$ matched load. The noted references contain additional details on the Mykonos LTD cavities [20] and the two-cavity module, Mykonos II [21-23], that preceded the Mykonos V module.

\footnotetext{
$\xi$ email: bthutse@sandia.gov
} 


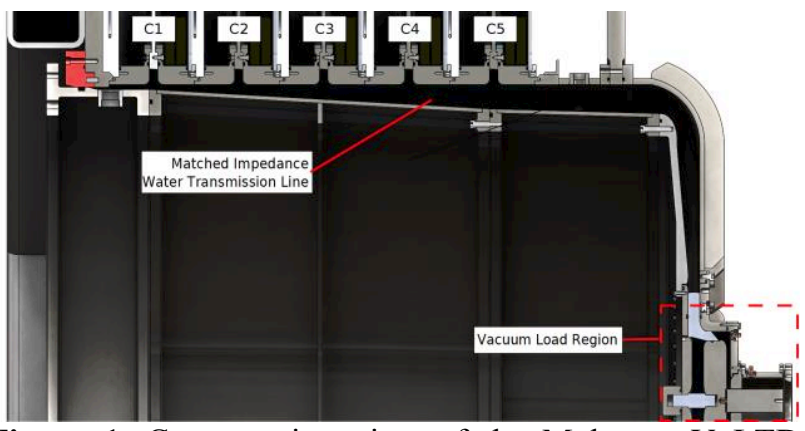

Figure 1. Cross-section view of the Mykonos V LTD Module.

\section{B. Load Hardware}

A vacuum transmission line load for Mykonos was designed and acquired for the vacuum power flow experiments. A cross-section view of the vacuum hardware is shown in Figure 2. A radial water transmission line, vacuum insulator, and radial vacuum transmission line served to direct power flow to the small diameter of the MITL being tested. The vacuum insulator serves to isolate the water-insulated transmission line from the vacuum-insulated transmission line. The vacuum insulator was also designed to direct power flow through a series water resistor. This water resistor was adjustable from approximately $0.1 \mathrm{ohm}$ to $0.5 \mathrm{ohm}$ and was used to dampen reflections back into the cavities from the shortcircuit inductive load.

The experiment MITL consisted of a small gap, $3 \mathrm{~cm}$ long, coaxial vacuum transmission line. The cathode radius is fixed at $3 \mathrm{~cm}$. The anode radius was varied to produce AK gaps between $1 \mathrm{~mm}$ and $1.3 \mathrm{~mm}$. A $15 \mathrm{nH}$ constant-inductance served as the load downstream of the experiment gap. The gap was designed to operate at fields of 1-2 MV/cm, well above the electron emission threshold of approximately $240 \mathrm{kV} / \mathrm{cm}$. The cathode radius of $3 \mathrm{~cm}$ was chosen to minimize the inductance of the vacuum region while still ensuring magnetic insulation would be established early in the pulse, at around $200 \mathrm{kA}$.

The experiment gap anode and cathode were made of stainless steel 304L. The anode and cathode were machined with no cutting oil and electropolished per ASTM B912 standards. After machining, the hardware was cleaned with isopropanol and a TX309 Texwipe, rinsed with isopropanol and blown dry with nitrogen, vacuum baked at $800 \mathrm{C}$ for four hours, then wrapped in a TX309 Texwipe and All-Foils UHV aluminum foil. The hardware was stored wrapped in the UHV foil until installed on the machine for use.

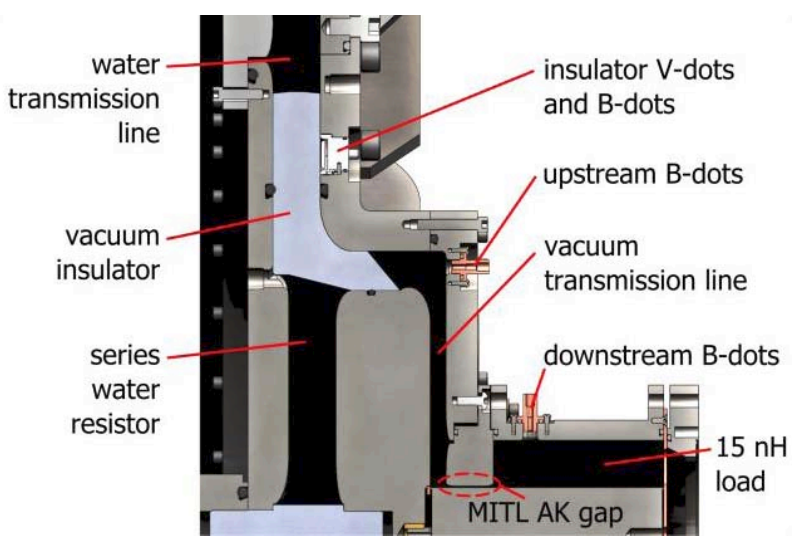

Figure 2. Cross-section view of the vacuum transmission line load hardware.

\section{Diagnostics}

The primary diagnostic included B-dot and D-dot monitors to measure current and voltage. Four B-dots and four D-dots were located at the vacuum insulator. These monitors were identical to those described in Sections III and $\mathrm{V}$ of [24]. In the vacuum transmission line, a total of eight B-dots were used to measure current. Four B-dots were located upstream and and four B-dots were located downstream of the experiment gap. The vacuum B-dots were designed as a 2-loop version of the inner-MITL current monitor described in Section IV of [24].

Prior to the experiments, the diagnostic monitors were calibrated in-situ. Two Pearson 3483 current monitors, accurate to $+/-1 \%$ absolute, were used in opposite polarities as calibration references for the vacuum and stack b-dot monitors. The negative polarity Pearson signal was inverted and averaged with the positive polarity Pearson signal to generate the final reference current signal. Voltage measured across a $2 \mathrm{k} \Omega$ resistor was used as the calibration reference for the D-dots. For calibration, the monitor signals were baseline-corrected to subtract any DC offset. The signals were then numerically integrated, and compared using an iterative routine to find the time shift and amplitude scale to minimize the pointwise rms differences between the reference waveform and the signal being calibrated. The B-dots also included a correction for the magnetic field penetration, as described in [24]. The signals gave rms point-wise deviations of a scaled signal divided by its peak of less than $1 \%$. Ten calibration tests were averaged to generate the gauge factors. The maximum shot-to-shot variation in gauge factors was $1.7 \%$ or less.

Vacuum diagnostics included two MKS 979b fullrange gauges to measure vacuum pressure upstream and downstream of the experiment gap. The reported vacuum pressure was measured by the upstream gauge. The upstream gauge was always the higher of the two vacuum measurements. This was due to a molecular pumping restriction caused by the experimental gap. The high upstream pressure was exacerbated by the vacuum seals on the insulator itself. A Stanford Research Systems RGA 
100 was also available to measure the vacuum composition during pumpdown.

\section{Shot Procedures}

The experiment gap anode and cathode were replaced for each shot series. All other vacuum hardware was reused, and refurbished if necessary. Vacuum surfaces that were coated with debris from breakdown of the experiment AK gap were refurbished with 3M ScotchBrite surface conditioning pads. The hardware was then vacuumed and wiped with a lint free cloth (TX309 Texwipe) and ethyl alcohol. The experiment gap anode and cathode hardware that were regularly replaced were not removed from the UHV foil they were stored in after processing until they were ready to install. The AK gap was aligned to $+/-2 \%$ of nominal gap using gauge pins to verify alignment. After the anode and cathode were aligned, the remaining vacuum hardware was installed and the system was pumped down. Pumpdown time was varied as an experiment variable. Typically the disassembly, refurbishment, and assembly of the load hardware were completed in approximately one hour. During installation, all vacuum hardware was handled only with clean nitrile gloves.

\section{III.EXPERIMENT RESULTS}

The current loss as a function of time for each experiment was determined from the B-dot diagnostics upstream and downstream of the MITL gap. The current measurements and the calculated loss current from a representative shot with the $1.0 \mathrm{~mm}$ gap are shown in Figure 3. Typically a small amount of loss could be measured early in the pulse prior to magnetic insulation. The loss would then hold constant, or in some cases decrease, until complete gap closure. Gap closure was indicated by a sharp divergence of the upstream and downstream current measurements. At this point the downstream current slowly L/R decays as magnetic energy stored in the load inductance dissipates.

\section{A. Anode-Cathode Gap}

The vacuum AK gap had the largest influence on the gap closure in the MITL. Experiments conducted with the $1.0 \mathrm{~mm}$ AK gap regularly resulted in complete gap closure at $40 \%-60 \%$ of peak current. In contrast, the 1.3 $\mathrm{mm}$ AK gap remained open during the current rise and did not close until at, or after, peak current. Figure 4 shows the measured loss current as a function of vacuum gap. It can be seen that the loss current decreases significantly as the vacuum gap increases from $1.0 \mathrm{~mm}$ to $1.3 \mathrm{~mm}$. The corresponding time to gap closure is shown in Figure 5. The time to gap closure increased from 50-80 ns with the $1.0 \mathrm{~mm}$ gap to $110-130 \mathrm{~ns}$ with the $1.3 \mathrm{~mm}$ gap. These times correspond to average gap closure velocities of approximately $1-2 \mathrm{~cm} / \mu \mathrm{s}$.

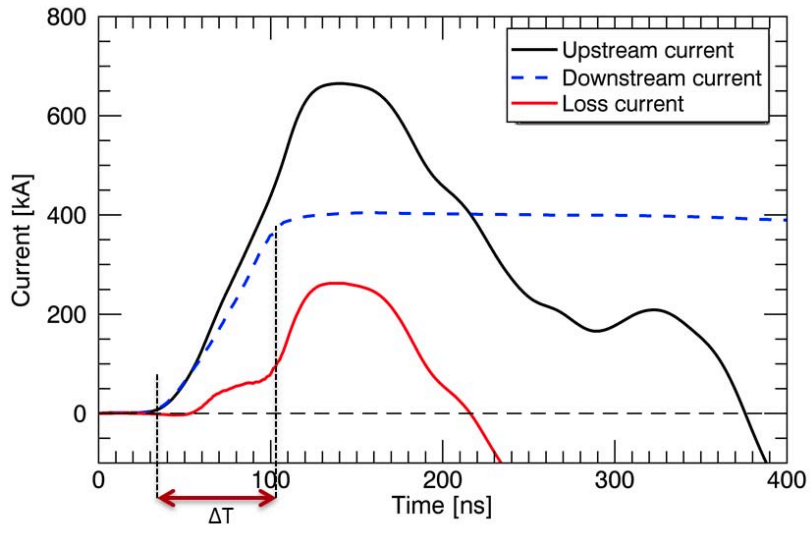

Figure 3. Representative current measurements taken upstream and downstream of the experiment AK gap. The loss current shown is the difference of the upstream and downstream measurements.

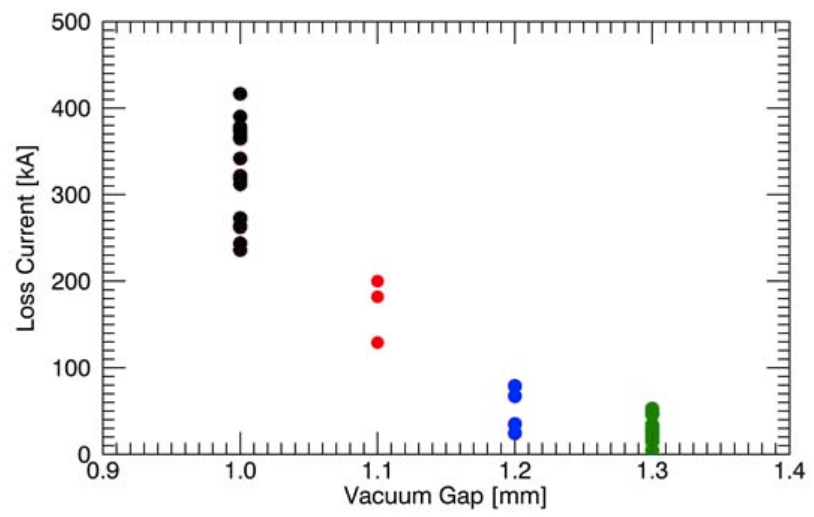

Figure 4. Loss current as a function of vacuum AK gap for $1.0,1.1,1.2$, and $1.3 \mathrm{~mm}$ gaps. Only shots taken at below $10^{-4}$ torr are shown.

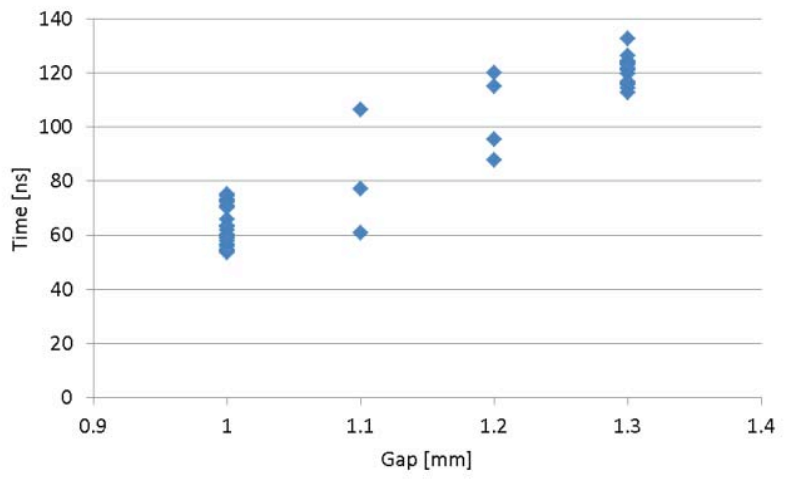

Figure 5. Time to gap closure as a function of vacuum AK gap.

\section{B. Vacuum pressure}

Loss current was found to be insensitive to the background gas pressure or the time spent under vacuum for pressures below rough vacuum as high as $10^{-3}$ torr. The loss current as a function of vacuum pressure for the 
1.0 and $1.3 \mathrm{~mm}$ gaps are shown in Figures 7 and 8 , respectively. The $1.0 \mathrm{~mm}$ gap was only tested at pressures between $10^{-3}$ torr and $10^{-6}$ torr. The time spent under vacuum ranged between 10 minutes and 14 days. The loss current was unaffected by either parameter and varied between approximately $200 \mathrm{kA}$ and $400 \mathrm{kA}$ for all shots. The $1.3 \mathrm{~mm}$ AK gap was tested over a larger pressure range, as high as $10^{-1}$ torr. The time to gap closure did not significantly decrease (resulting in an increased loss current) until the AK gap was operated at pressures above $10^{-2}$ torr. These high pressures correspond to collision mean free paths on the order of the vacuum AK gap resulting in enhanced breakdown of the AK gap.

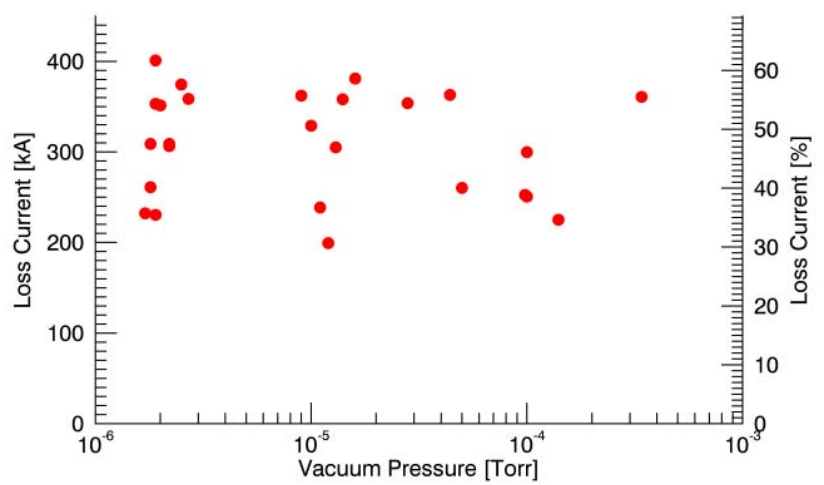

Figure 7. Loss current as a function of vacuum pressure for the $1.0 \mathrm{~mm} \mathrm{AK}$ gap. Percentage of loss current is based on a typical peak current of $650 \mathrm{kA}$ with negligible loss.

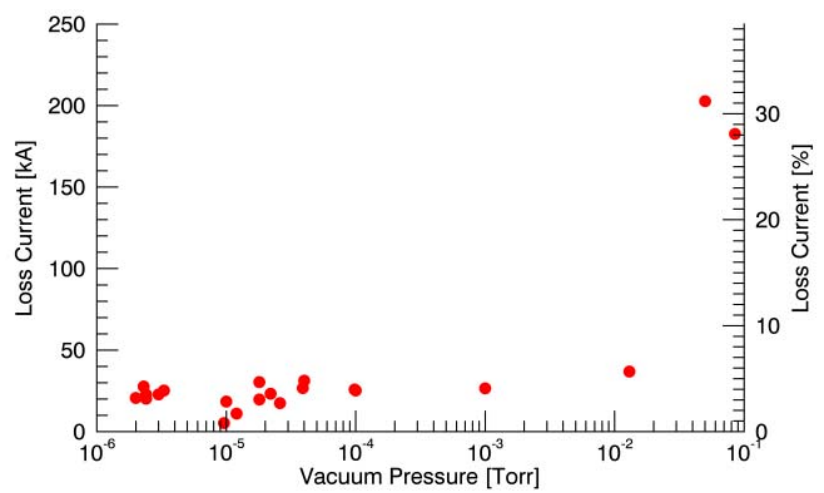

Figure 8. Loss current as a function of vacuum pressure for the $1.3 \mathrm{~mm} \mathrm{AK}$ gap. Percentage of loss current is based on a typical peak current of $650 \mathrm{kA}$ with negligible loss.

\section{IV.SUMMARY}

The experiments described represent the first phase of an effort to develop an improved understanding of current loss in vacuum transmission lines. In order to support this effort the Mykonos LTD accelerator was upgraded to a five cavity module and a vacuum transmission line load region was designed. Initial experiments conducted at low lineal current densities revealed the size of the vacuum gap had the largest influence on current loss. Increasing the gap from 1.0 to $1.3 \mathrm{~mm}$ delayed the vacuum gap closure until at, or after, peak current in the load. Additionally, it was concluded that in the limit of low lineal current density vacuum pressure had little effect on the measured loss current below approximately $10^{-4}$ torr. The loss current did not significantly increase until the background gas pressure increased above $10^{-2}$ torr, which corresponds to particle mean free paths on the order of the insulating vacuum gap.

\section{ACKNOWLEDGEMENTS}

Supported by the Laboratory Directed Research and Development program at Sandia National Laboratories, a multi-program laboratory managed and operated by Sandia Corporation, a wholly owned subsidiary of Lockheed Martin Corporation, for the U.S. Department of Energy's National Nuclear Security Administration under contract DE-AC04-94AL85000.

\section{REFERENCES}

[1] W. A. Stygar et al., "55-TW magnetically insulated transmission-line system: Design, simulations, and performance," Physical Review Special Topics Accelerators and Beams, vol. 12, no. 12, p. 120401, 2009, PRSTAB.

[2] T. D. Pointon and M. E. Savage, "2-D PIC Simulations of Electron Flow in the Magnetically Insulated Transmission Lines of Z and ZR," in Pulsed Power Conference, 2005 IEEE, 2005, Conference Proceedings, pp. 151-154.

[3] D. V. Rose et al., "Plasma evolution and dynamics in high-power vacuum-transmission-line post-hole convolutes," Physical Review Special Topics Accelerators and Beams, vol. 11, no. 6, p. 060401, 2008, PRSTAB.

[4] J. P. Martin et al., "Tailoring of electron flow current in magnetically insulated transmission lines," Physical Review Special Topics - Accelerators and Beams, vol. 12, no. 3, p. 030401, 2009, PRSTAB.

[5] J. M. Creedon, "Relativistic brillouin flow in the high mu/gamma diode," Journal of Applied Physics, vol. 46, no. 7, pp. 2946-2955, 1975.

[6] J. M. Creedon, "Magnetic cutoff in high current diodes," Journal of Applied Physics, vol. 48, no. 3, pp. 1070-1077, 1977.

[7] C. W. Mendel, D. B. Seidel, and S. E. Rosenthal, “A simple theory of magnetic insulation from basic physical considerations," Laser and Particle Beams, vol. 1, no. 03, pp. 311-320, 1983.

[8] W. A. Stygar et al., "Analytic model of a magnetically insulated transmission line with collisional flow 
electrons," Physical Review Special Topics - Accelerators and Beams, vol. 9, no. 9, p. 090401, 2006, PRSTAB.

[9] J. P. VanDevender, R. W. Stinnett, and R. J. Anderson, "Negative ion losses in magnetically insulated vacuum gaps," Applied Physics Letters, vol. 38, no. 4, pp. 229-231, 1981.

[10] R.W. Stinnett and T. Stanley, "Negative ion formation in magnetically insulated transmission lines," Journal of Applied Physics, vol. 53, no. 5, pp. 3819-3823, 1982.

[11] R. W. Stinnett et al., "Small gap experiments in magnetically insulated transmission lines," Plasma Science, IEEE Transactions on, vol. 11, no. 3, pp. 216219, 1983.

[12] R. Presura et al., "Operation regimes of magnetically insulated transmission lines," in Pulsed Power Conference, 2003. Digest of Technical Papers. PPC-2003. 14th IEEE International, vol. 2, 2003, Conference Proceedings, pp. 859-862 Vol.2.

[13] V. V. Ivanov et al., "Investigation of plasma evolution in a coaxial small-gap magnetically insulated transmission line," Plasma Science, IEEE Transactions on, vol. 32, no. 5, pp. 1843-1848, 2004.

[14] Y. L. Bakshaev et al., "Study of the dynamics of the electrode plasma in a high-current magnetically insulated transmission line," Plasma Physics Reports, vol. 33, no. 4, pp. 259-270, 2007.

[15] T. W. L. Sanford et al., "Measurement of electron energy deposition necessary to form an anode plasma in $\mathrm{Ta}$, Ti, and $\mathrm{C}$ for coaxial bremsstrahlung diodes," Journal of Applied Physics, vol. 66, pp. 10-22, 1989.

[16] J. Halbritter, "On contamination on electrode surfaces and electric field limitations," Electrical Insulation, IEEE Transactions on, vol. EI-20, no. 4, pp. 671-681, 1985.

[17] M. E. Cuneo, "The effect of electrode contamination, cleaning and conditioning on high-energy pulsed-power device performance," Dielectrics and Electrical Insulation, IEEE Transactions on, vol. 6, no. 4, pp. 469$485,1999$.

[18] W. A. Stygar, M. E. Savage, and M. C. Jones, "Analytic model of the Z vacuum system," 2013 Dec 6, Internal Memo, unpublished.

[19] A. Kim et al., "Prefire probability of the switch type Fast LTD," in Pulsed Power Conference, 2009. PPC '09. IEEE, 2009, Conference Proceedings, pp. 565-570.

[20] A. A. Kim et al., "Development and tests of fast 1MA linear transformer driver stages," Physical Review Special Topics - Accelerators and Beams, vol. 12, no. 5, p. 050402, 2009, PRSTAB.

[21] K. LeChien et al., "A 1-MV, 1-MA, 0.1-Hz linear transformer driver utilizing an internal water transmission line," in Pulsed Power Conference, 2009. PPC '09. IEEE, 2009, Conference Proceedings, pp. 1186-1191.

[22] M. G. Mazarakis et al., "Experimental validation of the first 1-MA water-insulated MYKONOS LTD voltage adder," in Pulsed Power Conference (PPC), 2011 IEEE, 2011, Conference Proceedings, pp. 625-628.
[23] M. E. Savage et al., "Temporally shaped current pulses on a two-cavity linear transformer driver system," in Pulsed Power Conference (PPC), 2011 IEEE, 2011, Conference Proceedings, pp. 844-849.

[24] T. C. Wagoner et al., "Differential-output B-dot and D-dot monitors for current and voltage measurements on a 20-MA, 3-MV pulsed-power accelerator," Physical Review Special Topics - Accelerators and Beams, vol. 11, no. 10, p. 100401, 2008, PRSTAB. 